\title{
BMJ Open Implementation of C-reactive protein point of care testing to improve antibiotic targeting in respiratory illness in Vietnamese primary care (ICAT): a study protocol for a cluster randomised controlled trial
}

\author{
Nga Thi Thuy Do (D) , ${ }^{1}$ Rachel Claire Greer (D) ,2,3 Yoel Lubell, ${ }^{2,3}$ \\ Sabine Dittrich (D) ${ }^{3,4}$ Maida Vandendorpe, ${ }^{4}$ Van Anh Nguyen, ${ }^{5}$ Pham Ngoc Thach, ${ }^{6}$ \\ Ta Thi Dieu Ngan, ${ }^{6}$ Nguyen Van Kinh, ${ }^{6}$ Cao Hung Thai, ${ }^{7}$ Le Thi Kim Dung, ${ }^{7}$ \\ Tu Nguyen Thi Cam, ${ }^{1}$ Thanh Ha Nguyen, ${ }^{1}$ Behzad Nadjm, ${ }^{1,3,8,9}$ \\ H Rogier van Doorn, ${ }^{1,3}$ Sonia Lewycka (1) 1,3,10
}

To cite: Thi Thuy Do N, Greer RC, Lubell Y, et al. Implementation of C-reactive protein point of care testing to improve antibiotic targeting in respiratory illness in Vietnamese primary care (ICAT): a study protocol for a cluster randomised controlled trial. BMJ Open 2020;10:e040977. doi:10.1136/ bmjopen-2020-040977

- Prepublication history and supplemental materials for this paper is available online. To view these files, please visit the journal online (http://dx.doi. org/10.1136/bmjopen-2020040977).

Received 27 May 2020 Revised 15 September 2020 Accepted 16 October 2020

Check for updates

(C) Author(s) (or their employer(s)) 2020. Re-use permitted under CC BY-NC. No commercial re-use. See rights and permissions. Published by BMJ.

For numbered affiliations see end of article.

Correspondence to Dr Nga Thi Thuy Do; ngadtt@oucru.org

\section{ABSTRACT}

Introduction C-reactive protein (CRP), a biomarker of infection, has been used widely in high-income settings to guide antibiotic treatment in patients presenting with respiratory illnesses in primary care. Recent trials in low- and middle-income countries showed that CRP testing could safely reduce antibiotic use in patients with non-severe acute respiratory infections (ARIs) and fever in primary care. The studies, however, were conducted in a research-oriented context, with research staff closely monitoring healthcare behaviour thus potentially influencing healthcare workers' prescribing practices. For policy-makers to consider wide-scale roll-out, a pragmatic implementation study of the impact of CRP point of care (POC) testing in routine care is needed.

Methods and analysis A pragmatic, cluster-randomised controlled trial, with two study arms, consisting of 24 commune health centres $(\mathrm{CHC})$ in the intervention arm (provision of CRP tests with additional healthcare worker guidance) and 24 facilities acting as controls (routine care). Comparison between the treatment arms will be through logistic regression, with the treatment assignment as a fixed effect, and the $\mathrm{CHC}$ as a random effect. With 48 clusters, an average of 10 consultations per facility per week will result in approximately 520 over 1 year, and 24960 in total (12480 per arm). We will be able to detect a reduction of $12 \%$ to $23 \%$ or more in immediate antibiotic prescription as a result of the CRP POC intervention. The primary endpoint is the proportion of patient consultations for ARI resulting in immediate antibiotic prescription. Secondary endpoints include the proportion of all patients receiving an antibiotic prescription regardless of ARI diagnosis, frequency of re-consultation, subsequent antibiotic use when antibiotics are not prescribed, referral and hospitalisation.

Ethics and dissemination The study protocol was approved by the Oxford University Tropical Research Ethics Committee (0xTREC, Reference: 53-18), and the ethical

\section{Strengths and limitations of this study}

This study will be conducted as a pragmatic implementation study of the impact of C-reactive protein (CRP) testing outside of the research context, to minimise disruption and alteration of routine care, and better inform wide-scale implementation of CRP testing in management of patients with acute respiratory infections in primary healthcare.

- A cluster randomised controlled trial design will prevent the contamination effect that might occur in an individual randomised controlled trial, where the same prescribers see patients in both intervention and control groups, and might also reduce antibiotic prescriptions for patients in the control group.

- A subsample of patients will be followed up over the phone and all patients' medical records will be reviewed to assess levels of re-consultations and admissions to hospital, providing important information regarding the safety and effectiveness of CRP testing in the real-life context of routine primary care in a resource constrained setting.

- In a setting like Vietnam, where antibiotics are commonly accessed without prescription outside the formal healthcare setting, to achieve maximal impact of CRP testing interventions on antibiotic consumption at community level, further work investigating the potential for point-of-care CRP testing in pharmacies and drug stores will be needed.

- Factors other than the study interventions may influence records of antibiotic prescribing, including other programmes in the area, reporting bias and the effects of the COVID-19 pandemic.

committee of the National Hospital for Tropical Diseases in Vietnam (Reference:07/HDDD-NDTW/2019). Results from this study will be disseminated via meetings with 
stakeholders, conferences and publications in peer-reviewed journals. Authorship and reporting of this work will follow international guidelines. Trial registration details NCT03855215; Pre-results.

\section{INTRODUCTION}

Antimicrobial resistance (AMR) is globally recognised as an important and growing problem in clinical settings, and Vietnam has AMR levels that are among the highest in the world. ${ }^{1}$ Reducing the inappropriate use of antibiotics has been shown to reduce the prevalence of antibiotic-resistant bacteria at the population-level in high-income countries, ${ }^{2}$ but few rigorously evaluated, population-level interventions aiming to tackle AMR by reducing unnecessary prescribing have been implemented and tested in low- and middleincome countries (LMICs). With $80 \%$ to $90 \%$ of human antibiotic consumption occurring in the community and the majority of these for patients with acute respiratory infections (ARIs), ${ }^{2-4}$ targeting interventions that can safely reduce antibiotic use in these cases could have a large impact on reducing overall antibiotic consumption.

Better targeting of antibiotics needs to start with simple tools to identify those patients presenting in the community who will not benefit from antibiotic treatment. C-reactive protein (CRP), a biomarker of inflammation, has been used for decades in high-income settings to guide this decision. ${ }^{5-8}$ A 2014 Cochrane review concluded that CRP was the best available biomarker to guide the use of antibiotics in ARIs. ${ }^{9}$ More recently a Vietnamese clinical trial showed that CRP testing could safely reduce antibiotic use in patients with mild ARIs in primary care in a LMIC. In this study, 2037 patients aged 1 to 65 years old were randomised to CRP point of care (POC) testing or routine care. Antibiotic prescription at first consultation in the CRP group was $43 \%$ compared with $63 \%$ in the routine care group ( OR $0.31,95 \%$ CI 0.34 to 0.49 ; $\mathrm{p}<0 \cdot 0001)$. The duration of symptoms between the groups did not vary and few hospital admissions occurred (6/901 in the CRP group and 8/874 in the control group).${ }^{10}$ A recent clinical trial in Thailand and Myanmar of 2410 febrile adults and children in whom the majority presented with respiratory symptoms, similarly found that CRP testing reduced antibiotic prescribing in primary care settings without affecting clinical outcomes $(39 \%$ in control group compared with $36 \%$ in CRP group A, which used a threshold of $20 \mathrm{mg} / \mathrm{L}$ (adjusted OR (aOR) of 0.86 , $95 \%$ CI 0.70 to $1.06, \mathrm{p}=0.17$ ), and $34 \%$ in CRP group B which used a threshold of $40 \mathrm{mg} / \mathrm{L}$ to guide antibiotic prescription (aOR of $0.80,95 \%$ CI 0.65 to $0.98, \mathrm{p}=0.03$ ) ${ }^{11}$

With extensive patient follow-up and in the presence of experienced research staff, these clinical trials provided important information regarding the safety and effectiveness of CRP testing in the context of primary care in Southeast Asia. The studies, however, were conducted in a research-oriented context, with research staff closely monitoring healthcare behaviour and thus potentially influencing healthcare workers' prescribing practices (Hawthorne effect). ${ }^{12}$ Furthermore, the CRP tests used in these studies required desktop hardware for analysis, affecting affordability and scalability in primary care in many LMIC settings. For policy-makers to consider wide-scale implementation of CRP testing, what is most needed is a pragmatic implementation study of the impact of CRP testing in routine care, with no research staff on site, using well-validated and commercially available lateral flow tests suitable for use in low-level facilities by less skilled personnel. Administering and interpreting such tests, which are similar to malaria rapid diagnostic tests that transformed the community management of malaria, is simple and safe using capillary blood obtained from a finger-prick.

\section{Rationale}

In line with Vietnamese Ministry of Health (MoH) improvement programmes in enhancing capacity of grassroots level, the proposed study will be monitored and evaluated as a quality improvement project-the introduction of commercially available lateral flow CRP tests with international regulatory approval, as a routine care service to improve the management of patients with ARI and reduce unnecessary antibiotic prescribing. The study aims to address the research question, namely can POC CRP tests introduced in routine primary healthcare reduce prescription of antibiotics for patients with ARIs, outside of the research context. Recent $\mathrm{MoH}$ guidelines for healthcare services in primary care will be integrated into training materials for local healthcare workers before implementation. ${ }^{13}$ No research staff will be present, and to minimise disruption and alteration of routine care, a waiver of written patient informed consent has been obtained from the relevant ethical review boards, in accordance with the 2016 WHO/CIOMS International Guidance for Health-related Research Involving Humans. ${ }^{14}$ Instead, patients in intervention clusters will be provided with information concerning how the test can assist healthcare workers in identifying when antibiotics are required, after which they will be free to refuse its use. Patients who verbally agree to use the CRP test will be asked to sign in a logbook at study sites.

A systematic review based on studies from hospital settings suggested that CRP levels $<20 \mathrm{mg} / \mathrm{L}$ provided the best rule-out value for serious infections in febrile children. ${ }^{15}$ Previous trials evaluating use of POC tests for patients with ARIs in high-income countries used an algorithm with a CRP cut-off of $<10 \mathrm{mg} / \mathrm{L}^{16}$ or $<20 \mathrm{mg} / \mathrm{L}$ to encourage prescribers to withhold antibiotic prescription. ${ }^{5} 817 \quad 18$ A clinical trial in Vietnam with patients with ARI used a threshold of $10 \mathrm{mg} / \mathrm{L}$ in children and $20 \mathrm{mg} / \mathrm{L}$ in adults to delay antibiotic prescription. ${ }^{10}$ These trials use quantitative readers which are unlikely to be affordable in resource constrained settings. In this study, we use the semi-quantitative test with three thresholds of $10 \mathrm{mg} / \mathrm{L}, 40 \mathrm{mg} / \mathrm{L}$ and $80 \mathrm{mg} / \mathrm{L}$. An algorithm with a CRP cut-off of $<10 \mathrm{mg} / \mathrm{L}$ will be used to recommend prescribers to wait and see (no antibiotics are recommended) for patients with ARI. The test selected for use in the study is the Actim CRP test from Medix Biochemica (Finland; ISO certification ISO13485:2016). 
The test is a simple lateral flow device that uses capillary blood, obtained through a finger/heel puncture. The test provides a semi-quantitative indication of whether CRP concentrations are $<10 \mathrm{mg} / \mathrm{L}$, between 10 to $40 \mathrm{mg} / \mathrm{L}$, between 40 to $80 \mathrm{mg} / \mathrm{L}$ or above $80 \mathrm{mg} / \mathrm{L}$, in under $5 \mathrm{~min}$ with minimal training requirements. The test has been approved by the European regulatory body (CE (Cetification Europe)-marking) as well as in 13 other countries around the world (including Switzerland, Norway, Iceland, Israel and Thailand) and has been validated for accuracy in previous publications. ${ }^{19} 20$ Our own research group has also confirmed the tests' accuracy in both laboratory and field environments, including their thermostability at room temperature in a tropical climate. ${ }^{21}$ Considering five lateral flow CRP tests which are either CE or FDA (Food and Drug Administration) marked and quote received, three tests (1.7US\$, $1.7 \mathrm{US} \$$ and $7 \mathrm{US} \$$ per test, respectively) providing low thresholds of 1,3 and $10 \mathrm{mg} / \mathrm{L} ; 5$ and $10 \mathrm{mg} / \mathrm{L} ; 3$ and $10 \mathrm{mg} / \mathrm{L}$, respectively, which are not eligible for our cutoff criteria. The other two are CE marked including CRP Rapid Test Cassette, Screen Italia, costs $0.9 €$ per test with threshold of 10 and $60 \mathrm{mg} / \mathrm{L}$ and Actim CRP Rapid Test (Medix Biochemia) with price of $2.5 €$ per test and thresholds of 10, 40 and $80 \mathrm{mg} / \mathrm{L}$. Finally, we chose Actim CRP test from Medix Biochemica with higher price but preferable cut-offs.

\section{Objectives}

Primary objective

The primary objective of this study is to assess the impact of CRP POC testing on the proportion of patients aged 1 to 65 years with ARI prescribed an initial antibiotic in routine primary healthcare.

\section{Secondary objectives}

The secondary objectives are to assess the consultation and prescription rates for patients with ARI aged 1 to 65 years in the preceding year, overall antibiotic prescription in all patients with ARI and those attending for non-routine visits in both study arms during the 1-year intervention, health workers' adherence to guidelines regarding indications for using the CRP test and CRP test results to guide antibiotic prescription, referral rates in ARI patients aged 1 to 65 years, subsequent antibiotic use within 2 weeks of follow-up, duration of symptoms, re-consultation rate within 30 days of attendance, hospitalisation within 2 weeks of attendance, the acceptability of CRP testing among healthcare workers and cost-effectiveness of the intervention compared with routine care.

\section{METHODS AND ANALYSIS}

We used the SPIRIT (Standard Protocol Items: Recommendations for Interventional Trials) reporting guidelines for clinical trials. ${ }^{22}$

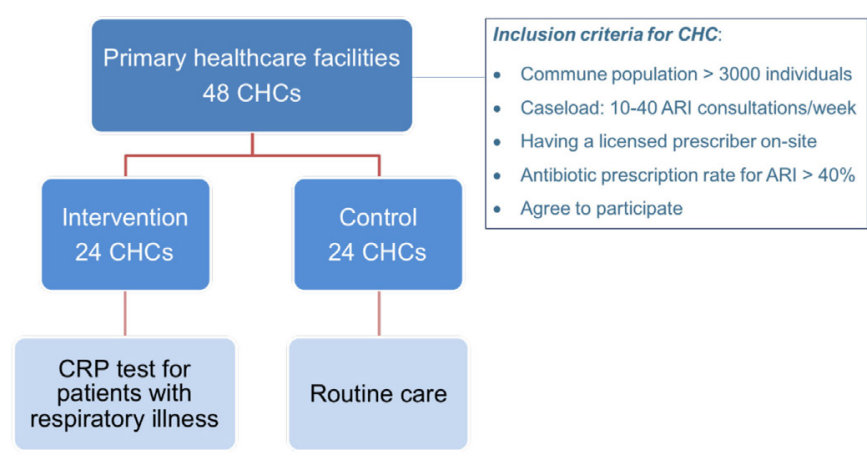

Figure 1 Study design - a cluster randomised control trial. ARI, acute respiratoryinfection; $\mathrm{CHCs}$, commune healthcentres; CRP, C-reactive protein.

\section{Study design}

The study will be conducted for 1 year (from June 2020 to May 2021) to allow for seasonal variations in ARI and prescribing. The intervention will be evaluated through a pragmatic, cluster-randomised controlled trial, with two study arms, consisting of 24 commune health centres (CHCs) in the intervention arm (provision of CRP tests with additional healthcare worker (HCW) guidance (initial training and desk reminder on the value of the test in guiding antibiotic prescription), and posters about test for both HCW and patients) and 24 CHCs acting as controls (routine care) (figure 1). Commune is a thirdlevel administrative subdivision of Vietnam. Communes have a lower status than provincial cities, towns or districts. ${ }^{23}$

\section{Study setting}

In the Vietnamese healthcare system, CHCs deliver most primary care services and national targeted health programmes to the population, especially in rural and mountainous areas, including hygiene, vaccinations, antenatal care, safe delivery and health education. Screening, examination, treatment and referrals for patients are also provided. In terms of coverage in Vietnam, 99\% of communes have a CHC, $70 \%$ of these have a doctor; others have an assistant doctor who is a licensed presriber. ${ }^{24}$

The study will be conducted in 48 CHCs in three rural districts (Truc Ninh, Nam Truc and Y Yen) in Nam Dinh, a northern province of Vietnam (figure 2). This province is approximately $90 \mathrm{~km}$ from Hanoi, the capital city.

\section{Eligibility criteria}

For CHCs

CHCs that (1) serve a commune population larger than 3000 individuals; (2) have an average caseload of between 10 to 40 consultations per facility per week for ARI verified by checking the database system for the preceding year; (3) have a licensed prescriber; and (4) have an electronic database for reporting patient level data, are considered eligible for being selected to conduct the intervention. CHCs that have an antibiotic prescription rate for ARI of below $40 \%$ are considered as outliers and not typical of routine care. ${ }^{10}$ 


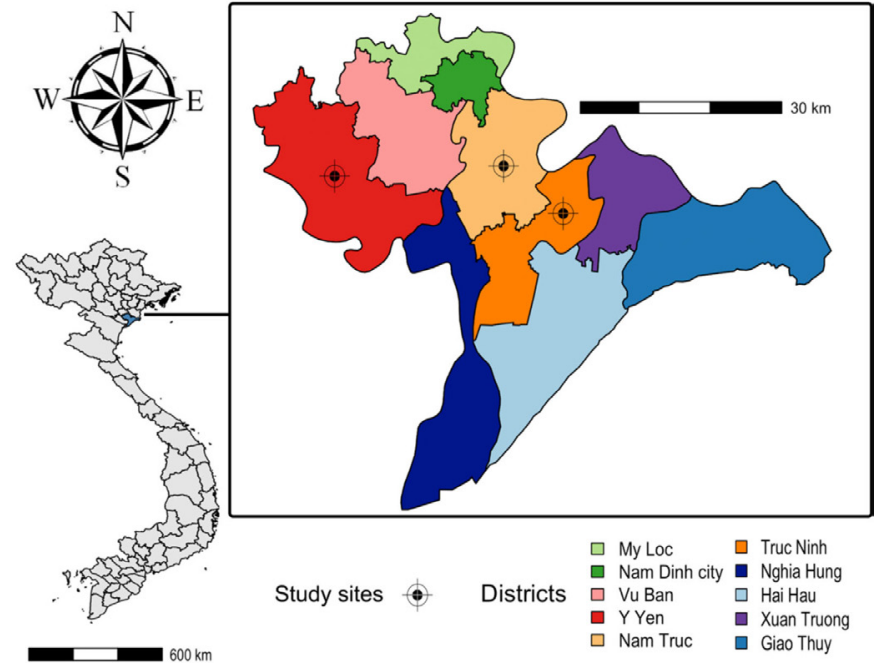

Figure 2 Map of Nam Dinh province where the intervention is being conducted. Loc, location.

\section{For patients}

Healthcare workers will be advised that use of the test should be restricted to patients within a recommended target population. The target population includes patients aged $\geq 1$ and $<65$ years old: ${ }^{25} 26$ having a health insurance (HI) number to enable data linkage with district hospital's database; visiting the CHC for a first consultation within 30 days with an ARI (as diagnosed by the healthcare worker); with at least one focal sign or symptom; and symptoms lasting less than 7 days. Focal signs and symptoms include (1) cough, (2) rhinitis (sneezing, nasal congestion or runny nose), (3) pharyngitis (sore throat), (4) shortness of breath, (5) wheezing, (6) chest pain or (7) auscultation abnormalities. The test will not be recommended in patients having a non-respiratory tract illness or patient with clinical signs of severity requiring referral to a higher-level facility based on the healthcare worker's clinical assessment. ${ }^{132}$ Data for patients with a HI number is routinely recorded and reported. We will use this data to assess trial outcomes including hospitalisations, thus only patients with health insurance numbers will be included in analyses (although HCWs in the intervention arm will be free to use the tests in patients without a HI number). Approximately $80 \%$ of patients at CHCs have HI. ${ }^{28}$

\section{Intervention}

CRP test

The Actim CRP Rapid Test (Medix Biochemica) will be made available to the HCWs at the intervention CHCs for use in patients in the target population. Printed guidance will be issued for the performance and interpretation of the CRP test results in terms of antibiotic-guided treatment. The treating HCW will decide, based on their clinical evaluation, whether or not to comply with this guideline. This guidance will also be discussed during the training sessions.
As the purpose of the study is to establish whether antibiotic prescription rates can be reduced by using CRP POC test results, we recommend that the HCWs use the CRP tests in all patients in the recommended target population. In the absence of danger signs of severity, if the CRP test result is below the $10 \mathrm{mg} / \mathrm{L}$ cut-off, no antibiotics are recommended (wait and see). If the CRP test result falls between $10 \mathrm{mg} / \mathrm{L}$ and $40 \mathrm{mg} / \mathrm{L}$, antibiotics are unlikely to be needed but should be considered in cases of high clinical concern (patients that are considered to be at higher risk of complications include patients who are systemically very unwell, patients with a pre-existing comorbidity, those currently using oral corticosteroids or those with a history of congestive heart failure). ${ }^{29}$ Antibiotics according to local guidelines are recommended if the CRP level is above $40 \mathrm{mg} / \mathrm{L}$.

Based on the previous clinical trial of CRP testing in ARI in primary healthcare facilities in Vietnam, we anticipate approximately $75 \%$ of patients to have CRP levels below $10 \mathrm{mg} / \mathrm{L}, 20 \%$ between 10 to $40 \mathrm{mg} / \mathrm{L}$ and $5 \%$ above $40 \mathrm{mg} / \mathrm{L}^{10}$

\section{Healthcare worker training}

Before the CHCs are randomised, we will present an overview of the study to all local HCWs and provide education about the role of antibiotics, antimicrobial resistance, clinical assessment and management of ARIs and identification of clinical signs of severity as well as good clinical practice for research.

Additional training will be provided to HCWs in the intervention arm. Findings from previous CRP studies have been used to inform the training and guidance for HCWs. Face-to-face training will be provided on how the test could be used to inform their prescribing behaviour, and a practical session on CRP testing. Content will include: which patients should be offered CRP tests; CRP cut-off values; safety nets and caution in result interpretation (including danger signs as described in Intergrated Management of Childhood Illness (IMCI) ${ }^{30} 31$ and Intergrated Management of Adolescent and Adult Illness (IMAI) ${ }^{32}$ guidelines and for patients with comorbidities). HCWs will be advised that the CRP cut-offs are guidelines and that antibiotics can be prescribed or withheld according to their clinical judgement. If the HCW feels the patient requires referral to a higher-level facility this should be done without delay, and the CRP tests should not be used in these cases. The training will be provided by the study team in the local language. The CHCs and HCWs will be given a telephone number to contact should any queries arise during the study. Laminated posters and desk reminders with the CRP cut-off values will be given to the intervention sites.

\section{Patient education}

Patients with ARIs in all CHCs will be provided with an information leaflet regarding rational use of antibiotics in ARI, and the implications of overuse of antibiotics for the development of AMR. An additional section for the 
intervention sites will explain the role of CRP testing to guide antibiotic prescriptions. Based on the given information, patients (or legally authorised representatives) in the intervention arm will freely decide whether they agree to do CRP test. Patients will be asked for verbal consent and to sign the CHC's logbook.

\section{Allocation and blinding}

The 48 CHCs will be randomly allocated to one of the two arms, stratified by district, and by baseline prescription level. Stratified random allocation will be done in Stata 14, using the randtreat command, with leftover CHCs (misfits) being allocated to maintain balance between strata. Due to the nature of the intervention, blinding of health-workers and patients to their allocation is not possible, though analysts and trial monitors will be blinded to the study allocation until the definitive analysis is performed.

\section{Outcomes}

The primary and secondary outcome measures for this study are shown in box 1 .

\section{Sample size}

We will use data on prescription rates for the preceding year from the $\mathrm{CHC}$ electronic system to assess variability between clusters, and randomly allocate CHCs to either the intervention or routine care arm. If there are substantial differences between clusters, we will consider stratification, or excluding clusters with extreme values (beyond the exclusion criterion for CHCs of antibiotic prescription rates below $40 \%$ ). Sample size calculations are based on probabilities of 0.05 and 0.2 for Type I and Type II errors, respectively (ie, $80 \%$ power), and inter-cluster coefficients of variation $(\mathrm{k})$ between 0.15 and 0.3 . The intracluster correlation coefficient (ICC) of 0.11 found in the previous trial in Vietnam, ${ }^{10}$ is equivalent to $\mathrm{k}=0.25$ ). The immediate antibiotic prescription rate in the control arm of the previous individually-randomised CRP trial in Vietnam was $64 \%$, with a reduction to $44 \%$ in the intervention arm (a $30 \%$ relative reduction). ${ }^{10}$

At each CHC, patients presenting with an ARI will be documented as normal in facility logbooks. For the analysis we will include data for patients with HI to enable data linkage with district hospital's database for checking subsequent hospitalisation; these represent more than $80 \%$ of the CHC attendees. ${ }^{28}$ With 24 clusters per arm (48 in total), an average of 10 consultations of patients with HI per facility per week will result in approximately 520 over 1 year, and 12480 per arm (24960 in total). We will be able to detect a reduction of $12 \%$ to $23 \%$ or more in immediate antibiotic prescription as a result of the CRP POC intervention (the range is dependent on the ICCthe degree of variability between clusters).

For each CHC, 15 patients consulting during a specified period will be interviewed via phone call by CHC nurses at four time points spread over a 1-year period, synchronised across all CHCs. With 1440 2-week exit interviews per arm (2880 in total) we will also be able to detect a reduction of $15 \%$ to $24 \%$ or more in overall antibiotic use as a result of the CRP POC intervention. To estimate the proportion with subsequent antibiotic use and test the

Box 1 Primary and secondary outcome measures

\section{Primary outcomes}

Proportion of patients aged 1 to 65 years, consulting for acute respiratory infection (ARI), who are prescribed initial antibiotics in the two study arms.

\section{Secondary outcomes}

a. The proportion of patients aged 1 to 65 years that consulted for ARI in the year preceding the study.

b. Proportion of patients aged 1 to 65 years, consulting for ARI, who were prescribed an antibiotic at the initial consultation in the year preceding the study.

c. Indications for C-reactive protein (CRP) test use including diagnosis, age, gender and the proportion of patients in whom use of the test was recommended that received the test.

d. Proportion of patients prescribed an immediate antibiotic in the intervention arm with:

a. $C R P<10 \mathrm{mg} / \mathrm{L}$.

b. $10 \mathrm{mg} / \mathrm{L} \leq \mathrm{CRP} \leq 40 \mathrm{mg} / \mathrm{L}$.

c. $\mathrm{CRP}>40 \mathrm{mg} / \mathrm{L}$.

e. Proportion of patients receiving an immediate antibiotic with the denominator being:

a. All attendances.

b. All non-routine attendances.

f. Proportion of patients receiving an immediate antibiotic prescription by diagnosis, age, season, recorded fever and sex.

g. The proportion of patients with ARI aged 1 to 65 years referred to a higher level facility at the initial consultation by checking the edatabase of health insurance $(\mathrm{HI})$ reimbursement or logbook at commune health centre $(\mathrm{CHC})$.

h. The proportion of patients with ARI aged 1 to 65 years who received subsequent antibiotics for any reason within 2 weeks of follow-up, verified by random subgroup patient exit interview via phone call follow-up after 14 days by CHC staff using follow-up questionnaire (online supplemental file 1). Subsequent antibiotics are defined as any antibiotic use within 2 weeks of follow-up excluding initial antibiotic prescriptions at $\mathrm{CHC}$.

i. Duration of ARI symptoms (verified by random patient exit interview via phone call follow-up after 2 weeks by $\mathrm{CHC}$ staff).

j. Proportion of patients with ARI aged 1 to 65 years who re-attend the health facility within a 30-day period, and whether antibiotics were prescribed comparing intervention $\mathrm{CHCs}$ and controls.

k. Proportion of eligible patients with ARI aged 1 to 65 years who are hospitalised within a 2-week period (verified by accessing provincial and district hospitals' database and random patient exit interview via phone call follow-up after 2 weeks by CHC staff) excluding patients referred at initial consultation.

I. Measure of usability and acceptability: pre-intervention and postintervention knowledge-attitude-practice (KAP) surveys will be conducted with all healthcare workers in both the intervention and control arms for their KAP on antibiotic use and antimicrobialresistance (AMR). Healthcare workers in the intervention arm will also be asked whether the test was usable and useful and whether they support their continued use by semi-structured interview guides.

m. Measure of cost-effectiveness: A cost analysis will be carried out to assess the budget implications for introducing the tests, accounting for the cost of treatments and referrals, and a cost-benefit analysis will compare any incremental costs with the modelled costs of AMR averted. 
follow-up procedure, 360 patients per arm (720 in total) will be interviewed in the lead-in period ( 1 month before the intervention). The previous CRP trial conducted in Vietnam did not find differences in prescribing for children and adults, ${ }^{6}$ but we will have sufficient power to detect substantial differences between subgroups if they exist. We would be able to detect a reduction in antibiotic use in subgroups of between 7 ( $1 \%$ of study population) and 90 (17\% of study population) patients per cluster-that is, the best-case scenario is a $30 \%$ reduction in antibiotic prescriptions and $\mathrm{k}=0.25 \quad(\mathrm{ICC}=0.11$ ) (as in the previous CRP study), the worst-case scenario is a $23 \%$ reduction and $\mathrm{k}=0.3(\mathrm{ICC}=0.16)$. We would have less than $80 \%$ power to detect a reduction smaller than $23 \%$ in a subgroup if the intercluster coefficient of variation is as high as or higher than 0.3. We considered including co-primary objectives as safety endpoints, but based on the previous CRP study in Vietnam, ${ }^{6}$ we believe that the absolute risk of an adverse outcome (ARI re-attendance, hospitalisation or death) for patients consulting for mild ARI is minimal. In our previous study in Vietnam there was a $1 \%$ hospitalisation rate, and a $0.3 \%$ to $0.5 \%$ re-attendance rate. Based on these figures, we estimate that we will be able to detect an absolute difference in adverse outcomes (hospitalisation or re-attendance) of $0.7 \%$ (from $1.5 \%$ to $2.2 \%$ ), with between $80 \%$ and $95 \%$ power. We anticipate a possible increase in immediate hospital referrals in patients with high CRP values.

\section{Data management}

\section{Data collection}

Quantitative data will be collected from all patients with HI attending the selected CHCs for a period of 1 year through routine CHC logbooks. Each patient will be assigned a unique study number which will be used in data entry and the database in order to protect the patient's identity. The study number will be made up of a CHC code ( 1 to 48 relating to the different CHCs), month (1 to 12 ) and sequential number in the CHC logbook (relating to the order of patient examination at each CHC). Electronic records containing data from the logbooks will be collected periodically throughout the study period, and used to create an electronic study database (REDCap). Patients' names and full addresses will not be included. All collected information will be anonymised and encrypted and data will be stored on secure computers only accessed by study investigators and data managers. Routine logbook data from all CHCs for the year preceding the study will be captured in order to identify any baseline variability in prescription rates for ARI patients, and to determine the number of patients meeting the target population for CRP testing.

The number of ARI consultations without CRP test results will also be collected from the patient screening logbook to evaluate the proportion of eligible population that do not receive CRP tests and associated reasons. As a safety endpoint, we will capture hospitalisation rates. We will collect admission data from the e-databases for

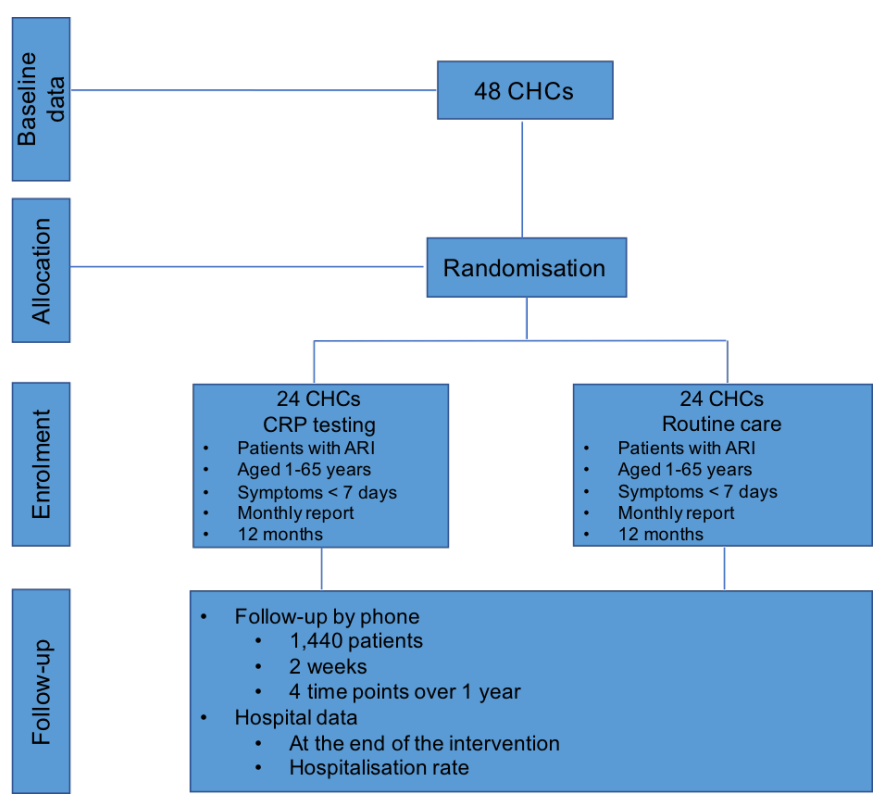

Figure 3 Trial flow diagram. ARI, acute respiratoryinfection; $\mathrm{CHCs}$, commune healthcentres; CRP, C-reactive protein.

HI reimbursement of the three district hospitals, for any patients who attended study CHCs in the previous month. Data will be linked using patients' HI numbers. Data requested will include the dates of admissions and diagnoses within 30 days of visiting the CHC. In addition, for each CHC, 15 patients consulting during a specified period will be interviewed via phone call by CHC nurses at four time points spread over a 1-year period, synchronised across all CHCs, excluding once in lead-in period (total 2880 patients) (figure 3). This supplementary data is to test if there is any inconsistency in safety endpoints compared with data from provincial and district hospital's database, as well as to collect data on subsequent antibiotic use. After data linkage, HI numbers will be dropped to protect patient anonymity.

In addition to data collection to assess the intervention's impact on the primary outcomes, we will conduct preintervention and post-intervention knowledge-attitudepractice (KAP) surveys with all HCWs in both arms to compare their KAP on antibiotic use and AMR before and after intervention. HCWs in the intervention arm will be interviewed by semi-structured interview guides to assess usability and acceptability of CRP POC testing and, and a cost analysis to measure the cost-effectiveness of the intervention will also be performed.

\section{Data coding}

A list of diagnoses or descriptions of ARIs will be compiled using the retrospective data from the year preceding the start of the study; this will be done prior to the analysis of the primary outcome. Where possible diagnoses will be grouped into conditions such as acute tonsillitis or acute sinusitis. ARIs will also be grouped into upper and lower respiratory tract infections. ICD-10 (InternationalClassification of Diseases, $10^{\text {th }}$ Revision) codes reported in the HI database will also be used to define ARI consultations. 
Any patient who re-attends the same CHC within 30 days of their initial visit will be classed as a repeat attendance, regardless of the secondary diagnosis. This means that we will be able to see the frequency of re-attendances and whether this is affected by use of the CRP test. It will also allow us to see whether antibiotics are initially withheld and then given at a subsequent visit (acknowledging the limitation that we may class some patients as re-attenders when their subsequent visit is actually for a new problem or illness).

\section{Data analysis plan}

Data will be analysed by intention-to-treat, such that all patients aged 1 to 65 , consulting for ARI, will be included in the analysis. The primary endpoint of this trial is the proportion of patient consultations for ARI resulting in immediate antibiotic prescription. The primary comparison between the treatment arms will be a logistic regression with the treatment assignment as a fixed effect, and the $\mathrm{CHC}$ as a random effect. A two-sided $95 \%$ CI for the OR of receiving antibiotic prescription rate (primary endpoint) will be calculated. Measures of baseline prescribing will be included in the model in order to adjust for any imbalances in randomisation at the outset.

The primary endpoint (proportion of patients with ARI with immediate antibiotic prescription) will also be investigated in subgroups to assess whether the reduction in antibiotic prescribing is homogeneous across subgroups. Specifically, intervention effects and appropriate interaction tests for heterogeneity will be calculated in the following predefined subgroups: age (adults vs children defined as $<16$ years-old according to age cut-off used in Vietnam), sex, proportion of patients in whom the test was recommended that received the test, $\mathrm{CHC}$, febrile versus non-febrile patients with ARI, CRP level, diagnosis, prescriber and seasonality.

The proportion of all patients receiving an antibiotic prescription (regardless of ARI diagnosis), frequency of re-consultation, duration of symptoms, subsequent antibiotic use within 2 weeks, referral at initial consultation and hospitalisation within 2 weeks of follow-up (verified by accessing provincial and district hospitals' database and patient exit interview via phone call follow-up after 2 weeks by CHC staff) will be compared between the two arms in the same way as the primary endpoint. In the subset of patients for whom a follow-up phone call will be conducted, time to resolution of symptoms will be visualised using Kaplan-Meier curves, and formal comparisons between the two treatment groups will be based on the Cox proportional hazards model with the treatment assignment and the age stratum as fixed effects and the CHC as a Gaussian random effect.

We will also conduct per-protocol analyses, including only patients with ARI in intervention CHCs who were given a CRP test. Data collected during the 12 months prior to intervention onset means that we will be able to look at overall changes in outcome indicators over time. Factors other than the study interventions may influence records of antibiotic prescribing, including other programmes in the area, reporting bias and the effects of the COVID-19 pandemic. Interim analysis while the study is ongoing will not be done as our trial poses no more than minimal risks to participants. We do not foresee any adverse effects of the intervention, which has previously been shown to be safe, so we do not intend to apply stopping rules. ${ }^{1033}$

\section{Access to data}

After entry into the electronic data environment, data will be uploaded to a central database. Access to data will be password protected, and only accessible to study investigators and sponsors for remote monitoring. Source data will be kept for 10 years according to local authority's requirement.

\section{Data handling and record keeping}

Quantitative data will be captured electronically from patient record databases routinely maintained with data from health station logbooks. Identifiers such as patients' names and full address will not be included. Patients will be assigned a unique composite ID number for the study, and HI numbers will be used to link patients making multiple visits. The name and any other address detail below the commune level will not be included in any study data electronic files. HI numbers will be dropped after linkage with hospital databases. Data will be stored electronically on a central server at OUCRU (Oxford UniversityClinical Research Unit).

\section{Monitoring}

As the research poses no more than minimal risks to participants, a data and safety monitory board will not be required. Based on previous studies we anticipate no serious adverse events associated with the CRP tests and patients will not be followed up, except for a subsample of patients who will be followed up by phone by CHC staff, 2 weeks after the initial consultation. We will retrospectively request data on hospital admissions for patients with ARI in both arms. This will be done at the end of the study. Other than the finger prick sample for the CRP test no samples will be taken or stored. CRP test kits and other consumables will be re-stocked on a monthly basis and checked to confirm they are properly used for study purposes only. Healthcare workers in both intervention and routine care arms will receive monthly supervision visits to improve and monitor the quality of record keeping.

\section{ETHICS AND DISSEMINATION}

The protocol (V.1.5, 04 November 2019) has been approved by the Oxford University Tropical Research Ethics Committee (OxTREC, Reference number: 53-18), and the ethical committee of the National Hospital for Tropical Diseases in Vietnam (Reference:07/HDDDNDTW/2019). Permission to carry out this study was obtained from local authorities.

A waiver of written informed consent from patients (or that of a legally authorised representative) to participate in the study was obtained from the relevant ethical review 
boards, to ensure as little disruption to routine practice as possible. This is in accordance with the 2016 CIOMS Guideline $10^{14}$ which states that in the following circumstances a waiver of informed consent could be applied: (1) if the research would not be feasible or practicable to carry out without the waiver or modification; (2) if the research has important social value; and (3) if the research poses no more than minimal risks to participants. The proposed study and intervention meet all these criteria. The research question relates to the impact of CRP testing in a routine care environment, therefore extensive informed consent procedures by research staff or the local HCWs on site could alter prescribing behaviour. This would directly affect the primary outcome and make us unable to answer our research question because we would no longer be operating in a routine care environment. Sim and Dawson argue that waiving informed consent is justifiable when the methodological integrity of the study is brought into question. ${ }^{34}$ Taking written informed consent would also disrupt patient care. In order for healthcare systems to be responsive and improve patient care implementation, studies are required to generate evidence for policy change and to ensure that the benefits seen in research settings are translated into routine care. The research question is of high importance and social value, with ARIs and febrile illness being the leading causes of often unnecessary antibiotic prescribing in the community, a key driver of AMR. The use of CRP testing may also help to identify those patients who need antibiotics but may be missed by routine clinical assessment. Lastly, CRP testing in these patients is of minimal risk, already widely used for these purposes in other settings and with large clinical trials with extensive patient follow-up previously conducted in Southeast Asian primary care settings, including in Vietnam. The drawing of finger prick blood is also of minimal risk, and the blood taken is only being used for direct patient care. Other than the finger prick sample for the CRP test no samples will be taken or stored.

Instead, patients in intervention clusters will be provided with information concerning how the test can assist HCWs in identifying when antibiotics are required. Patients who verbally agree to use the CRP test will be asked to sign a logbook at the study sites (online supplemental file 2). Similar to other tests that may be indicated, patients or their legal guardians will be able to refuse the CRP test, without any other impact on their care. If they wish to decline the test, their autonomy will be respected.

If there are any ineligible patients enrolled, the study investigator will report a protocol violation to the local Institutional Review Board.

Written consent for HCW interviews will be obtained, after explaining the purpose of the interview, risks, benefits and other details.

Results will be used to inform the design of implementation strategies as well as policy-making decisions. Results will be written up for publication in peer-reviewed journals and communicated as part of scientific meetings, as appropriate and as agreed by all members of the study team.
Author affiliations

${ }^{1}$ Oxford University Clinical Research Unit, Hanoi, Vietnam

${ }^{2}$ Mahidol Oxford Tropical Medicine Research Unit, Bangkok, Thailand

${ }^{3}$ Nuffield Department of Medicine, University of Oxford, Oxford, UK

${ }^{4}$ Malaria/Fever Program, Foundation for Innovative New Diagnostics (FIND), Geneva, Switzerland

${ }^{5}$ Foundation for Innovative New Diagnostics (FIND), Hanoi, Vietnam

${ }^{6}$ National Hospital of Tropical Diseases, Hanoi, Vietnam

${ }^{7}$ Medical Services Administration, Ministry of Health, Hanoi, Vietnam

${ }^{8}$ University College London Hospitals NHS Foundation Trust, London, UK

${ }^{9}$ Clinical Services Department, MRC Unit The Gambia at The London School of Hygiene, Banjul, Gambia

${ }^{10}$ University of Auckland, Auckland, New Zealand

\section{Twitter Nga Thi Thuy Do @Nga Thuy}

Contributors YL, SL and SD were responsible for conception and funding application. YL, RCG, SD, SL, NTTD and HRvD were in charge of study design. MV, VAN and BN contributed to the study protocol development. PNT, TTDN, NVK, CHT and LTKD were responsible for local Institutional Review Board approval, getting permission from local authorities and training for sites. TNTC and THN are responsible for coordinating study activities across sites. HRvD and SL supervise the study implementation. SL and NTTD will lead data analysis. Drafting of study protocol manuscript was prepared by NTTD and RCG. All authors contributed to the final revision and approved the submission.

Funding This work was supported by FIND, Geneva, Switzerland, with funds from the Australian government, and UK aid from the UK government, grant number F017-0015.

Map disclaimer The depiction of boundaries on the map(s) in this article does not imply the expression of any opinion whatsoever on the part of BMJ (or any member of its group) concerning the legal status of any country, territory, jurisdiction or area or of its authorities. The map(s) are provided without any warranty of any kind either express or implied.

Competing interests We declare no competing interests. Neither Medix Biochemica nor any of its distributors, have had involvement with the funding or design of this study. The investigators have no financial connection to this or any other CRP test manufacturer or distributor.

Patient consent for publication Not required.

Provenance and peer review Not commissioned; externally peer reviewed.

Supplemental material This content has been supplied by the author(s). It has not been vetted by BMJ Publishing Group Limited (BMJ) and may not have been peer-reviewed. Any opinions or recommendations discussed are solely those of the author(s) and are not endorsed by BMJ. BMJ disclaims all liability and responsibility arising from any reliance placed on the content. Where the content includes any translated material, BMJ does not warrant the accuracy and reliability of the translations (including but not limited to local regulations, clinical guidelines, terminology, drug names and drug dosages), and is not responsible for any error and/or omissions arising from translation and adaptation or otherwise.

Open access This is an open access article distributed in accordance with the Creative Commons Attribution Non Commercial (CC BY-NC 4.0) license, which permits others to distribute, remix, adapt, build upon this work non-commercially, and license their derivative works on different terms, provided the original work is properly cited, appropriate credit is given, any changes made indicated, and the use is non-commercial. See: http://creativecommons.org/licenses/by-nc/4.0/.

\section{ORCID iDs}

Nga Thi Thuy Do http://orcid.org/0000-0002-3435-1402

Rachel Claire Greer http://orcid.org/0000-0001-9327-0234

Sabine Dittrich http://orcid.org/0000-0002-4522-2788

Sonia Lewycka http://orcid.org/0000-0002-5923-9468

\section{REFERENCES}

1 Kaya Z, Küçükcongar A, Vurallı D, et al. Leukocyte populations and C-reactive protein as predictors of bacterial infections in febrile outpatient children. Turk J Haematol 2014;31:49-55.

2 Goossens H, Ferech M, Vander Stichele R, et al. Outpatient antibiotic use in Europe and association with resistance: a cross-national database study. Lancet 2005;365:579-87. 
3 Shallcross LJ, Davies DSC. Antibiotic overuse: a key driver of antimicrobial resistance. Br J Gen Pract 2014;64:604-5.

4 Greer RC, Intralawan D, Mukaka M, et al. Retrospective review of the management of acute infections and the indications for antibiotic prescription in primary care in northern Thailand. BMJ Open 2018;8:e022250.

5 Cals JWL, Butler CC, Hopstaken RM, et al. Effect of point of care testing for $\mathrm{C}$ reactive protein and training in communication skills on antibiotic use in lower respiratory tract infections: cluster randomised trial. BMJ 2009;338:b1374.

6 Cals JWL, Chappin FHF, Hopstaken RM, et al. C-reactive protein point-of-care testing for lower respiratory tract infections: a qualitative evaluation of experiences by GPs. Fam Pract 2010;27:212-8.

7 Cals JWL, de Bock L, Beckers P-JHW, et al. Enhanced communication skills and C-reactive protein point-of-care testing for respiratory tract infection: 3.5-year follow-up of a cluster randomized trial. Ann Fam Med 2013;11:157-64.

8 Little P, Stuart B, Francis N, et al. Effects of internet-based training on antibiotic prescribing rates for acute respiratory-tract infections: a multinational, cluster, randomised, factorial, controlled trial. Lancet 2013;382:1175-82.

9 Aabenhus R, Jensen J-US, Jørgensen KJ, et al. Biomarkers as point-of-care tests to guide prescription of antibiotics in patients with acute respiratory infections in primary care. Cochrane Database Syst Rev 2014;15.

10 NT D, NT T, Tran NT, et al. Point-Of-Care C-reactive protein testing to reduce inappropriate use of antibiotics for non-severe acute respiratory infections in Vietnamese primary health care: a randomised controlled trial. Lancet Glob Health 2016;4:e633-41.

11 Althaus T, Greer R, Swe M, et al. Randomised controlled trial of point-of-care $\mathrm{C}$-reactive protein testing on antibiotic prescription in febrile patients attending primary care in Southeast Asia. Lancet Global Health. In Press.

12 McCambridge J, Witton J, Elbourne DR. Systematic review of the Hawthorne effect: new concepts are needed to study research participation effects. J Clin Epidemiol 2014;67:267-77.

13 Ministry of Health. Guidelines for examination and treatment at commune health centres [Vietnamese], 2014. Available: https:// extranet.who.int/ncdccs/Data/VNM_D1_QD so 2919_QD-BYT huong dan kham chua benh tram y te xa.pdf

14 World Health Organisation. International ethical guidelines for health-related research involving humans. Geneva: WHO Press, 2016. https://cioms.ch/wp-content/uploads/2017/01/WEB-CIOMSEthicalGuidelines.pdf

15 Van den Bruel A, Thompson MJ, Haj-Hassan T, et al. Diagnostic value of laboratory tests in identifying serious infections in febrile children: systematic review. BMJ 2011;342:d3082.

16 Diederichsen HZ, Skamling M, Diederichsen A, et al. Randomised controlled trial of CRP rapid test as a guide to treatment of respiratory infections in general practice. Scand J Prim Health Care 2000;18:39-43.

17 Cals JWL, Schot MJC, de Jong SAM, et al. Point-of-care Creactive protein testing and antibiotic prescribing for respiratory tract infections: a randomized controlled trial. Ann Fam Med 2010;8:124-33.

18 Andreeva E, Melbye $\mathrm{H}$. Usefulness of C-reactive protein testing in acute cough/respiratory tract infection: an open cluster-randomized clinical trial with C-reactive protein testing in the intervention group. BMC Fam Pract 2014;15:80.

19 Evrard B, Roszyk L, Fattal S, et al. [Evaluation of rapid, semiquantitative assay of C-reactive protein in whole blood, Actim CRP]. Ann Biol Clin 2005;63:525-9.

20 Brouwer N, van Pelt J. Validation and evaluation of eight commercially available point of care CRP methods. Clin Chim Acta 2015;439:195-201.

21 Lubell Y, Blacksell SD, Dunachie S, et al. Performance of C-reactive protein and procalcitonin to distinguish viral from bacterial and malarial causes of fever in Southeast Asia. BMC Infect Dis 2015;15.

22 Chan A-W, Tetzlaff JM, Altman DG, et al. SPIRIT 2013 statement: defining standard protocol items for clinical trials. Ann Intern Med 2013;158:200-7.

23 General Statistics Office of Vietnam. Administrative units [Vietnamese], 2018. Available: http://www.gso.gov.vn/dmhc2015/ TongHop.aspx

24 Ministry of Health. Vietnam health service delivery profile. Vietnam: Develop in collaboration between WHO and the Misnistry of Health, 2012. http://www.wpro.who.int/health_services/service_delivery_ profile_vietnam.pdf

25 McNally M, Curtain J, O’Brien KK, et al. Validity of British thoracic Society guidance (the CRB-65 rule) for predicting the severity of pneumonia in general practice: systematic review and meta-analysis. Br J Gen Pract 2010;60:e423-33.

26 Lemiengre MB, Verbakel JY, Colman R, et al. Point-Of-Care CRP matters: normal CRP levels reduce immediate antibiotic prescribing for acutely ill children in primary care: a cluster randomized controlled trial. Scand J Prim Health Care 2018;36:423-36.

27 World Health Organisation. Integrated management of adolescent and adult illness. Geneva: WHO Press, 2004. http://www.who.int/hiv/ pub/imai/acute_care.pdf

28 Thao NM. Vietnam social insurance conference: implementing duties in 2017 [in Vietnamese], 2016. Available: http:// baobaohiemxahoi.vn/vi/tin-chi-tiet-bhxh-viet-nam-to-chuc-hoinghicai-cach-hanh-chinh-va-trien-khai-nhiem-vu-nam-2017-96cf7794. aspx

29 National Institute for Health and Care Excellence. Respiratory tract infections (self-limiting): prescribing antibiotics, 2019. Available: https://www.nice.org.uk/guidance/cg69/resources/respiratory-tractinfections-selflimiting-prescribing-antibiotics-pdf-975576354757

30 WHO. Integrated management of childhood illness. Geneva, Switzerland, 2014.

31 Senn N, Rarau P, Salib M, et al. Use of antibiotics within the IMCl guidelines in outpatient settings in Papua New Guinean children: an observational and effectiveness study. PLoS One 2014;9:e90990.

32 The World Health Organization. Integrated management of adolescent and adult illness: interim guidelines for first-level facility health workers at health centre and district outpatient clinic: acute care. Rev 3, 2009.

33 Althaus T, Greer RC, Swe MMM, et al. Effect of point-of-care Creactive protein testing on antibiotic prescription in febrile patients attending primary care in Thailand and Myanmar: an open-label, randomised, controlled trial. Lancet Glob Health 2019;7:e119-31.

$34 \mathrm{Sim}$ J, Dawson A. Informed consent and cluster-randomized trials. Am J Public Health 2012;102:480-5. 